\title{
Warming Up Before a 2o-Minute Endurance Effort: Is It Really Worth It?
}

Barranco-Gil D, Alejo LB, Valenzuela PL, Gil-Cabrera J, Montalvo-Pérez

A, Talavera E, Moral-González S, Clemente-Suárez VJ, Lucia AABSTRACT

ABSTRACT:

PURPOSE: To analyze the effects of different warm-up protocols on endurance-cycling performance from an integrative perspective (by assessing perceptual, neuromuscular, physiological, and metabolic variables). METHODS: Following a randomized crossover design, 15 male cyclists (35 [9] y; peak oxygen uptake [VO2peak] 66.4 [6.8] $\mathrm{mL} \cdot \mathrm{kg}-1 \cdot \mathrm{min}-1$ ) performed a 20-minute cycling time trial (TT) preceded by no warm-up, a standard warm-up (10 min at 60\% of VO2peak), or a warm-up that was intended to induce potentiation postactivation (PAP warm-up; 5 min at $60 \%$ of VO2peak followed by three 10-s all-out sprints). Study outcomes were jumping ability and heart-rate variability (both assessed at baseline and before the TT), TT performance (mean power output), and perceptual (rating of perceived exertion) and physiological (oxygen uptake, muscle oxygenation, heart-rate variability, blood lactate, and thigh skin temperature) responses during and after the TT. RESULTS: Both standard and PAP warm-up (9.7\% [4.7\%] and 12.9\% [6.5\%], respectively, $P<.001$ ), but not no warm-up (-0.9\% [4.8\%], $P=.074)$, increased jumping ability and decreased heart-rate variability (-7.9\% [14.2\%], $P=.027 ;-20.3 \%$ [24.7\%], $P=.006 ;$ and $-1.7 \%$ [10.5\%], $P=.366)$. Participants started the TT (minutes $O-3$ ) at a higher power output and oxygen uptake after PAP warm-up compared with the other 2 protocols $(P<.05)$, but no betweenconditions differences were found overall for the remainder of outcomes ( $P$ > .05). CONCLUSIONS: Compared with no warm-up, warming up enhanced jumping performance and sympathetic modulation before the $T T$, and the inclusion of brief sprints resulted in a higher initial power output during the TT. However, no warm-up benefits were found for overall TT performance or for perceptual or physiological responses during the TT. 


\section{KEYWORDS:}

cycling; exercise; preconditioning; time trial 